\title{
Reduced susceptibility to carbapenems in Klebsiella pneumoniae clinical isolates associated with plasmid-mediated $\beta$-lactamase production and OmpK36 porin deficiency
}

\author{
Xuan Ding Wang, Jia Chang Cai, Hong Wei Zhou, Rong Zhang \\ and Gong-Xiang Chen \\ 2nd Affiliated Hospital of Zhejiang University, Zhejiang University, 88 Jiefang Road, Hangzhou \\ 310009, PR China
}

Correspondence

Gong-Xiang Chen

chengong218@163.com

Received 17 November 2008

Accepted 12 May 2009

\section{INTRODUCTION}

Klebsiella pneumoniae is one of the most important pathogens that causes nosocomial infections. Due to the extensive use of extended-spectrum cephalosporins, extended-spectrum $\beta$-lactamase (ESBL)-producing $K$. pneumoniae has become an increasingly serious problem. Carbapenems are commonly used to treat serious infections caused by multidrug-resistant Gram-negative bacteria, especially strains producing high levels of AmpC cephalosporinases or ESBLs. Although carbapenem-resistant $K$. pneumoniae is uncommon, a number of $K$. pneumoniae isolates have been reported to be resistant to carbapenems mediated by metallo- $\beta$-lactamases in Singapore, Brazil, Turkey and Taiwan, including IMP-1 and IMP-8 (Aktas et al., 2006; Koh et al., 1999; Lincopan et al., 2005; Yan et al., 2001). Recently, the number of $K$. pneumoniae isolates producing KPC-type carbapenemases

Abbreviations: ESBL, extended-spectrum $\beta$-lactamase; i.v., intravenously; OMP, outer-membrane protein; pl, isoelectric point. has increased dramatically (Smith Moland et al., 2003; Wei et al., 2007; Woodford et al., 2004; Yigit et al., 2001).

A combination of high-level production of AmpC $\beta$ lactamases (e.g. ACT-1, CMY-4 and DHA-1) or SHV-2 together with porin loss can result in resistance or reduced susceptibility to carbapenems in K. pneumoniae (Bradford et al., 1997; Cao et al., 2000; Crowley et al., 2002; Lee et al., 2007a). Recently, an outbreak of imipenem-non-susceptible K. pneumoniae, caused by the production of CMY-2 and DHA-1 together with OmpK35 and OmpK36 porin loss, was reported in Korea (Lee et al., 2007b).

Studies have been conducted to elucidate the mechanism of resistance to carbapenems in K. pneumoniae as a result of a deficiency of porins in combination with the production of $\beta$-lactamases. Lee et al. (2007a) reported that strains that are deficient in both OmpK35 and OmpK36 have resistance to carbapenems in the presence of $\beta$-lactamases. However, in a strain where only OmpK35 was deficient and OmpK36 was expressed, the strain maintained its suscept- 
ibility regardless of the introduction of other $\beta$-lactamases such as DHA-1. In a separate study, Mena et al. (2006) reported that, in the presence of CTX-M-1, loss of both OmpK35 and OmpK36 resulted in resistance, and expression of OmpK36 alone resulted in the recovery of susceptibility. These findings suggest that OmpK36 plays an important role in resistance. However, all studies to date have been carried out in strains in which OmpK35 is also deficient.

We collected two carbapenem-non-susceptible K. pneumoniae isolates, Z2554 and Z2110, that did not produce any type of carbapenemase, although carbapenem-resistant Enterobacteriaceae isolated in our hospital are mainly due to the production of KPC-2 (Cai et al., 2008; Zhang et al., 2007, 2008), indicating that other carbapenem resistance mechanisms, such as changes in outer-membrane permeability combined with $\beta$-lactamase production or an efflux pump, might be involved. Further studies indicated that the two strains produced a number of $\beta$-lactamases. Examination of their outer-membrane proteins (OMPs) showed that both strains expressed OmpK35 but failed to express OmpK36. In addition, an unidentified protein of $\sim 26 \mathrm{kDa}$ was found in one of the strains. To the best of our knowledge, this is the first report of K. pneumoniae clinical isolates with reduced carbapenem susceptibility that produce OmpK35 but have no OmpK36 expression.

\section{METHODS}

Bacterial strains and antimicrobial susceptibility testing. Two carbapenem-non-susceptible K. pneumoniae strains, Z2554 and Z2110, were isolated from the sputum of two patients in November 2006 and identified using a Vitek system (bioMérieux). One patient was hospitalized in the oncology ward due to extensive abdominal cavity metastasis after a radical operation for gastric cancer. Cefoperazone/sulbactam and ciprofloxacin $[2.0 \mathrm{~g}$, intravenously (i.v.), twice a day] and ciprofloxacin ( 0.4 g, i.v., twice a day) were administered for pulmonary infection for 2 weeks and then replaced with cefminox ( $2.0 \mathrm{~g}$, i.v., twice a day) and gatifloxacin ( $0.4 \mathrm{~g}$, i.v., once a day). Two days later, K. pneumoniae Z2554 was isolated. The other patient was hospitalized in the respiratory ward for severe pneumonia and treated with multiple antimicrobials, including ceftriaxone ( $2.0 \mathrm{~g}$, i.v., once a day) and ciprofloxacin ( $0.5 \mathrm{~g}$, i.v., once a day) for 5 days, panipenem ( 1.0 g, i.v., every $12 \mathrm{~h})$ and mycomycin (500 $000 \mathrm{U}$, orally, three times a day) for 2 weeks, and cefminox ( $2.0 \mathrm{~g}$, i.v., twice a day) and gatifloxacin $(0.4 \mathrm{~g}$, i.v., once a day) for 5 days, after which $K$. pneumoniae Z2110 was isolated.

The MICs of 16 antimicrobial agents were determined using an agar dilution method according to Clinical and Laboratory Standards Institute recommendations (CLSI, 2006). The antimicrobial agents were: piperacillin, cefotaxime, gentamicin, tetracycline, sulfonamide (Sigma), imipenem, ertapenem, cefoxitin (Merck), meropenem (Dainippon Sumitomo Pharma), piperacillin/tazobactam (Wyeth Holdings), cefoperazone/sulbactam (Pfizer), ceftazidime (GlaxoSmithKline), ceftriaxone (Roche), aztreonam, cefepime (Bristol-Meyers Squibb) and ciprofloxacin (Bayer).

PFGE. PFGE of the two K. pneumoniae isolates was performed according to the protocols of PulseNet (http://www.cdc.gov/pulsenet/ protocols.htm) in a Rotaphor system 6.0 instrument (Whatman
Biometra). The $X b a \mathrm{I}$ restriction patterns of their genomic DNA were analysed and interpreted according to the criteria of Tenover et al. (1995).

Conjugation experiment. A conjugal transfer experiment was carried out in mixed broth cultures. Rifampicin-resistant Escherichia coli EC600 $\left(\mathrm{LacZ}^{-}, \mathrm{Nal}^{\mathrm{R}}\right.$, $\left.\mathrm{Ri}^{\mathrm{R}}\right)$ was used as the recipient. E. coli transconjugants were selected on Mueller-Hinton agar containing rifampicin $\left(700 \mu \mathrm{g} \mathrm{ml}^{-1}\right)$ and imipenem $\left(0.5 \mu \mathrm{g} \mathrm{ml}^{-1}\right)$, or rifampicin $\left(700 \mu \mathrm{g} \mathrm{ml}^{-1}\right)$ and cefoxitin $\left(16 \mu \mathrm{g} \mathrm{ml}^{-1}\right)$ (all from Sigma). The selected colonies were picked and identified using a Vitek system. Plasmid DNA of the E. coli transconjugant, K. pneumoniae clinical isolates and E. coli V517 (Macrina et al., 1978) were obtained using an AxyPrep plasmid miniprep kit (Axygen Scientific).

IEF of $\boldsymbol{\beta}$-lactamase. Crude $\beta$-lactamase preparations were obtained by ultrasonic treatment of bacterial cells. IEF was carried out on a PhastGel polyacrylamide gel ( $\mathrm{pH}$ 3-9; Amersham Biosciences) using a PhastSystem (Pharmacia Biotech) and the method of Mathew et al. (1975). $\beta$-Lactamase activity was visualized by staining the gel with nitrocefin (Oxoid). Isoelectric points (pIs) were determined after comparison with the known $\beta$-lactamases TEM-28 (pI 6.1), SHV-7 (pI 7.6) and ACT-1 (pI 9.0).

PCR amplification and DNA sequence analysis of bla genes. Plasmids from K. pneumoniae Z2554 and Z2110 and the E. coli transconjugant were used as templates. The primers used to amplify $b l a_{\mathrm{TEM}}, b l a_{\mathrm{CTX}-\mathrm{M}}$ and $b l a_{\mathrm{DHA}}$ were as described by Yan et al. (2002) and Yu et al. (2007). The reaction was conducted in a GeneAmp PCR system 9600 thermal cycler (Applied Biosystems). PCR products were purified and sequenced directly. DNA sequences were compared with reported nucleotide sequences in GenBank.

Analysis of OMPs. OMPs were obtained as described by HernándezAllés et al. (1999). Strains were grown in Mueller-Hinton broth overnight at $37{ }^{\circ} \mathrm{C}$ with shaking. OMP profiles were determined by SDS-PAGE using $11.6 \%$ acrylamide/ $0.4 \%$ bisacrylamide $/ 0.1 \%$ SDS gels $(0.75 \mathrm{~mm}$ thick). These mini-gels were run at a constant current of $20 \mathrm{~mA}$ for $85 \mathrm{~min}$ with a Mini Protein 3 slab electrophoresis cell (Bio-Rad). Bands were visualized by staining with Coomassie brilliant blue.

The ompK35 and ompK36 genes of K. pneumoniae Z2554 and Z2110 were amplified by PCR (Kaczmarek et al., 2006). The sequences were analysed by comparison with reported nucleotide sequences in GenBank.

$\mathbf{N}$-terminal sequence analysis. The $\mathrm{N}$-terminal sequence of the protein identified by SDS-PAGE of the OMPs from Z2110 was determined. The protein was transferred to a PVDF membrane after SDS-PAGE in a CAPS buffer system. The membrane was stained with Coomassie brilliant blue and the band corresponding to a protein of $\sim 26 \mathrm{kDa}$ was excised and used for $\mathrm{N}$-terminal amino acid sequence analysis. The amino acid sequence of the transferred protein was determined using the Edman method using an Applied Biosystems protein sequencer (Procise $492 \mathrm{cLC}$ ). The sequence of 15 aa was determined. The sequence was used to search and compare with sequences in GenBank

\section{RESULTS AND DISCUSSION}

\section{Antimicrobial agent susceptibility and PFGE profiles}

The MICs of 16 antimicrobial agents are presented in Table 1. K. pneumoniae Z2554 and Z2110 showed 
Table 1. Antimicrobial susceptibility patterns of the $K$. pneumoniae isolates and the $E$. coli transconjugant

\begin{tabular}{|c|c|c|c|c|}
\hline \multirow[t]{2}{*}{ Antimicrobial agent ${ }^{*}$} & \multicolumn{4}{|c|}{$\operatorname{MIC}\left(\mu \mathrm{g} \mathrm{ml}^{-1}\right)$} \\
\hline & K. pneumoniae Z2554 & K. pneumoniae Z2110 & $\begin{array}{l}\text { K. pneumoniae } \mathrm{Z} 2110 \\
\text { E. coli transconjugant }\end{array}$ & E. coli EC600 \\
\hline Imipenem & 0.5 & 4 & $\leqslant 0.125$ & $\leqslant 0.125$ \\
\hline Imipenem + CLA & 0.25 & 4 & NT & NT \\
\hline Imipenem + CLO & 0.5 & 0.25 & NT & NT \\
\hline Imipenem + CLA + CLO & $\leqslant 0.125$ & $\leqslant 0.125$ & NT & NT \\
\hline Meropenem & 8 & 2 & $\leqslant 0.125$ & $\leqslant 0.125$ \\
\hline Meropenem + CLA & 2 & 1 & NT & NT \\
\hline Meropenem + CLO & 8 & 0.5 & NT & NT \\
\hline Meropenem + CLA + CLO & 1 & 0.25 & NT & NT \\
\hline Ertapenem & 16 & 32 & $\leqslant 0.125$ & $\leqslant 0.125$ \\
\hline Ertapenem + CLA & 4 & 16 & NT & NT \\
\hline Ertapenem + CLO & 8 & 4 & NT & NT \\
\hline Ertapenem + CLA + CLO & 1 & 0.5 & NT & NT \\
\hline Piperacillin & $>256$ & $>256$ & 4 & 1 \\
\hline Piperacillin/tazobactam & 256 & 64 & 4 & 1 \\
\hline Cefoxitin & 32 & $>512$ & 32 & 8 \\
\hline Ceftazidime & 256 & 16 & 1 & 0.25 \\
\hline Cefotaxime & $>256$ & 128 & 0.25 & $\leqslant 0.125$ \\
\hline Ceftriaxone & $>256$ & 256 & $\leqslant 0.125$ & $\leqslant 0.125$ \\
\hline Cefoperazone/sulbactam & 256 & 64 & $\leqslant 0.125$ & $\leqslant 0.125$ \\
\hline Cefepime & $>256$ & 128 & $\leqslant 0.125$ & $\leqslant 0.125$ \\
\hline Aztreonam & $>256$ & 32 & $\leqslant 0.125$ & $\leqslant 0.125$ \\
\hline Ciprofloxacin & $>32$ & $>32$ & 0.5 & $\leqslant 0.125$ \\
\hline Gentamicin & 1 & $>256$ & $>256$ & 0.25 \\
\hline Tetracycline & 8 & 32 & NT & NT \\
\hline Sulfamethoxazole & 16 & 8 & NT & NT \\
\hline
\end{tabular}

NT, Not tested.

${ }^{\star}$ CLA, Clavulanic acid (concentration fixed at $4 \mu \mathrm{g} \mathrm{ml}^{-1}$ ); CLO, cloxacillin (concentration fixed at $200 \mu \mathrm{g} \mathrm{ml}{ }^{-1}$ ).

resistance to ertapenem and various levels of reduced susceptibilities to imipenem and meropenem. K. pneumoniae Z2554 was highly resistant to penicillins, cephalosporins, aztreonam and quinolones, but remained susceptible to aminoglycosides. K. pneumoniae Z2110 was resistant to all of the tested antimicrobial agents. The MIC of cefoxitin for K. pneumoniae Z2110 was much higher than that for Z2554, although the MICs of the $\beta$-lactamase inhibitors, cephalosporins and aztreonam were lower. The MICs against ertapenem, imipenem and meropenem were also determined in the presence of $\beta$-lactamase inhibitors. As shown in Table 1, the MICs were significantly reduced by these inhibitors.

K. pneumoniae Z2554 and Z2110 had different PFGE patterns (Fig. 1), indicating that they were genetically unrelated.

\section{Transfer of antimicrobial agent resistance and plasmid analysis}

For K. pneumoniae Z2554 and Z2110, no colonies grew on Mueller-Hinton agar containing rifampicin and imipenem.
Fig. 1. PFGE fingerprinting of $K$. pneumoniae $Z 2554$ and $Z 2110$ after Xbal digestion. Lanes: 1, Z2554; 2, Z2110. 
Transfer of cefoxitin resistance from K. pneumoniae Z2110 to E. coli EC600 was successful. The transfer frequency was approximately $10^{-5}-10^{-6}\left(60-100\right.$ transconjugants per $10^{7}$ recipients). The E. coli transconjugant was resistant to cefoxitin and gentamicin, and moderately resistant to penicillins, but was susceptible to cephalosporins, aztreonam, quinolones and carbapenems (Table 1).

The plasmid profiles (Fig. 2) indicated that $K$. pneumoniae Z2554 and Z2110 harboured several plasmids. The E. coli transconjugant acquired a plasmid of $\sim 50 \mathrm{~kb}$ from $K$. pneumoniae Z2110. This transfer into E. coli EC600 by conjugation resulted in resistance to cefoxitin and gentamicin, indicating that $b l a_{\mathrm{DHA}-1}$ and the aminoglycoside-resistant determinant were located on the same plasmid.

\section{IEF analysis}

IEF analysis of the two isolates and the E. coli transconjugant of Z2110 demonstrated that there were two $\beta$ lactamases in K. pneumoniae Z2554 with pIs of 5.4 and 7.9, whilst $K$. pneumoniae $\mathrm{Z} 2110$ produced three $\beta$-lactamases with pIs of 5.4, 7.8 and 7.9. The E. coli transconjugant produced a single $\beta$-lactamase of pI 7.8 (data not shown).

\section{PCR and DNA sequence analysis}

To confirm the genotypes of the $\beta$-lactamases detected by IEF, PCR was conducted for plasmids extracted from $K$. pneumoniae Z2554 and Z2110 and the E. coli transconjugant. As a result, $b l a_{\mathrm{TEM}}$ and $b l a_{\mathrm{CTX}-\mathrm{M}-9}$ group genes were detected in both clinical isolates, whilst the $b a_{\text {DHA }}$ gene was detected in $K$. pneumoniae Z2110 and the E. coli transconjugant. After comparing the DNA sequences with known genes in GenBank, we found that isolate Z2554 contained sequences encoding TEM-1 and CTX-M-14,

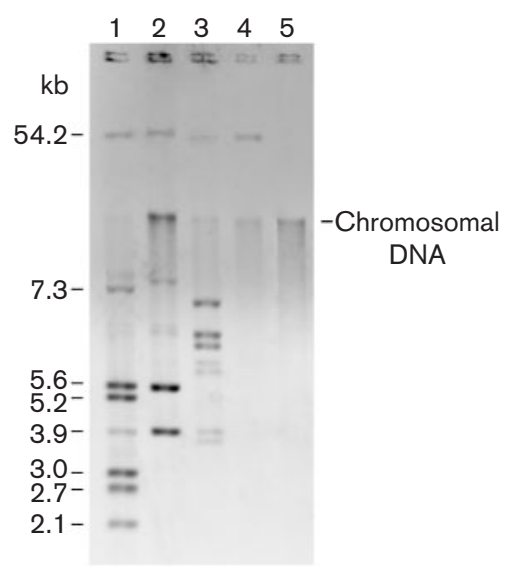

Fig. 2. Plasmid profiles of $K$. pneumoniae Z2554 and Z2110, and the E. coli transconjugant of Z2110. Lanes: 1, E. coli V517; 2, K. pneumoniae Z2554; 3, K. pneumoniae Z2110; 4, E. coli transconjugant of Z2110; 5, E. coli EC600 (negative control). whilst isolate Z2110 encoded an extra AmpC $\beta$-lactamase, DHA-1, and the E. coli transconjugant of Z2110 encoded a single DHA-1.

\section{SDS-PAGE and OMP-encoding gene analysis}

K. pneumoniae Z2554 and Z2110 showed various levels of resistance to carbapenems but did not produce any type of carbapenemase, indicating that other carbapenem resistance mechanisms, such as alteration in outer-membrane permeability or an efflux pump, might be involved. However, as the efflux mechanism is unlikely to be involved in the resistance of enterobacteria, we focused on an alteration in the OMPs. SDS-PAGE analysis of the OMPs was performed, and the result revealed loss of an OMP of $\sim 39 \mathrm{kDa}$ in both K. pneumoniae Z2554 and Z2110 compared with K. pneumoniae ATCC 13883 (Fig. 3). It was uncertain whether the $39 \mathrm{kDa}$ OMP was OmpK36 or OmpK35, as in some strains OmpK36 migrates faster than OmpK35 (Hernández-Allés et al., 1999). The ompK35 gene was amplified, sequenced and aligned with that of $K$. pneumoniae KT755 (GenBank accession no. AJ011501) (Doménech-Sánchez et al., 2003), and point mutations were found at nt 420, 474, 537 and 786 on the nucleotide sequence in both K. pneumoniae Z2554 and Z2110, and an additional point mutation was present in Z2554 at nt 249. However, point mutations in the ompK35 gene were all silent and did not lead to amino acid sequence changes. Comparing the ompK36 gene of K. pneumoniae Z2554 and Z2110 with that of K. pneumoniae C3 (GenBank accession no. Z33506; Albertí et al., 1995), several small DNA fragment insertions and a number of point mutations were observed. The sequence of the ompK36 gene from Z2110 and Z2554 is shown in Fig. 4. These mutations resulted in alteration of the ORF and disruption of the ompK36 gene. These results suggested that it was OmpK36 that was not expressed in K. pneumoniae Z2554 and Z2110.

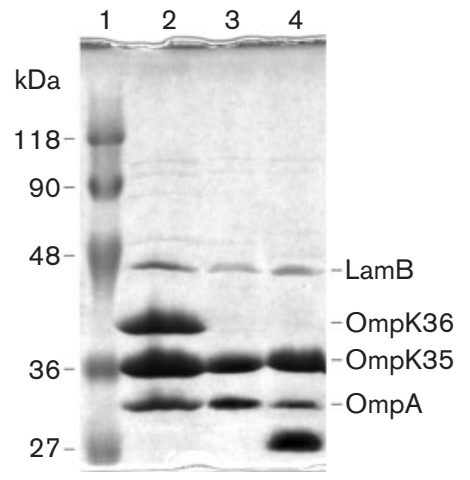

Fig. 3. SDS-PAGE analysis of the OMPs of $K$. pneumoniae isolates Z2554 and Z2110, and K. pneumoniae ATCC 13883. Lanes: 1, molecular mass standards (MBI Fermentas); 2, $K$. pneumoniae ATCC 13883; 3, K. pneumoniae Z2554; 4, K. pneumoniae Z2110. 


\begin{tabular}{|c|c|c|}
\hline pneumonia & 1 & GTTAAAGTACTGTCCCTCCTGGTACCGGCTCTGCTGGTAGCAGGCGCAGCAAATGCGGCTGAAATTTAT \\
\hline Protein & 1 & $\begin{array}{llllllllll} & \text { A } & \text { L } & \text { L } & \text { V }\end{array}$ \\
\hline K. pneumoniae $2110 / 2554$ & 76 & 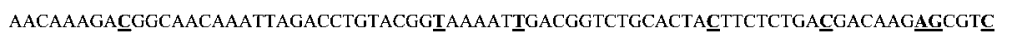 \\
\hline Protein & 26 & $\begin{array}{llllllll}Y & \mathbf{F} & \mathrm{S} & \mathrm{D} & \mathbf{D} & \mathrm{K} & \underline{\mathbf{s}} & \mathrm{V}\end{array}$ \\
\hline K. pneumoniae $2110 / 2554$ & 51 & GACGGCGACCAGACCTACATGCGTGTAGGCGTGAAAGGCGAAACCCAGATCAACGACCAGCTGACCGGTTACGGC \\
\hline Protein & 51 & $\begin{array}{lllllllllll} & \underline{\mathbf{V}} & \mathbf{G} & \mathrm{V} & \mathrm{K} & \mathrm{G} & \mathrm{E} & \mathrm{T} & \mathrm{Q} & \mathrm{I} & \mathrm{N}\end{array}$ \\
\hline K. pnetmoniae $2110 / 2554$ & 226 & CAGTGGGAATACAACGTTCAGGCGAACAACACTGAAAGCTCCAGCGATCAGGCATGGACTCGTCTGGCATTCGCA \\
\hline Protein & 76 & $\begin{array}{lllllllllllllllllllllllllll}Q & W & E & Y & N & V & Q & A & N & N & T & E & S & S & S & D & Q & A & W & T & R & \text { L } & A & F & A\end{array}$ \\
\hline K. pneumoniae $2110 / 2554$ & 301 & GGCCTGAAATTITGGCGACGCGGGCTCTTTCGACTACGGTCGTAACTACGGCGTAGTATACGACGTAACGTCCTGG \\
\hline Protein & 101 & $\begin{array}{lllllllllllll}A & G & S & F & D & Y & G & R & N & Y & G\end{array}$ \\
\hline K. pneumoniae 2110 & 376 & ACCGACGTTCTGCCGGAATTCGGCGGCGACACCTACGG TTCTGACAACTTCCTGCAGTCCCGTGCTAACGGCGTT \\
\hline K. pneumoniae 2554 & 376 & ACCGACGTTCTGCCGGAATTCGGCGGCGACACCTACGGTTTTCTGACAACTTCCTGCAGTCCCGTGCTAACGGCGTT \\
\hline Protein & 126 & $\begin{array}{llllllllllllll}T & D & V & L & P & E & F & G & G & D & T & Y & G\end{array}$ \\
\hline & 126 & $\begin{array}{llllllllllllllllllllllllllll}\mathrm{T} & \mathrm{D} & \mathrm{V} & \mathrm{L} & \mathrm{P} & \mathrm{E} & \mathbf{F} & \mathrm{G} & \mathrm{G} & \mathrm{D} & \mathrm{T} & \mathrm{Y} & \mathrm{G} & \mathrm{F} & \mathrm{L} & \mathrm{T} & \mathrm{T} & \mathrm{S} & \mathrm{C} & \mathrm{S} & \mathbf{P} & \mathrm{V} & \mathrm{L} & \mathrm{T} & \mathrm{A} & \mathbf{L}\end{array}$ \\
\hline K. pneumoniae 2110 & 451 & GCAACCTACCGTAACTCTGATTTCTTCGGTCTGGTTGACGGCCTGAACTTTGCTCTGCAGTATCAGGGIAAAAAC \\
\hline K. pneumoniae 2554 & 453 & GCAACCTACCGTAACTCTGATTCTTCGGTCTGGTTGACGGCCTGAACTTTGCTCTGCAGTATCAGGGTAAAAAC \\
\hline Protein & 151 & 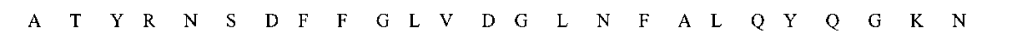 \\
\hline & 152 & L $\quad \mathrm{T} A *$ \\
\hline K. pneumoniae 2110 & 526 & 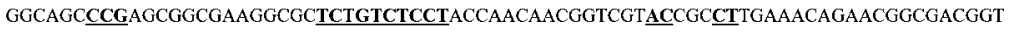 \\
\hline K. pneumonicue 2554 & 528 & TCTCCGACCAACAACGGTCGTGGCGCTCTGAAACAGAACGGIGACGGC \\
\hline Protein & 176 & 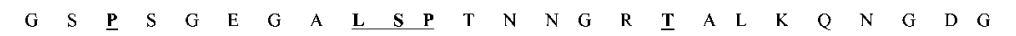 \\
\hline K. pneumoniae 2110 & 601 & 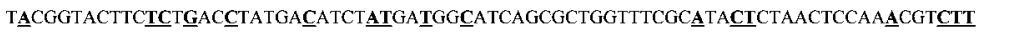 \\
\hline K. pneun & 600 & 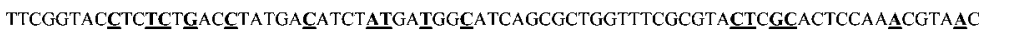 \\
\hline Protein & 201 & $\begin{array}{lllllllllllllllllllllllll}Y & G & T & S & \text { L } & \text { T } & Y & \text { D } & \text { l } & \text { Y } & \text { D } & \text { G } & \text { l } & \text { S } & \text { A } & \text { G } & \text { F } & \text { A } & \text { Y } & \text { S } & \text { N } & \text { S } & \text { K } & \text { R }\end{array}$ \\
\hline K. pneumoniae 2110 & 676 & GGCGACCAGAACGCAAGCTGGCACTGGGTCGTGGCGACAACGCTGAAACCTACACCGGCGGTCTGAAATACGACG \\
\hline K. pnezmoniue 2554 & 675 & TGGATAAAGGCCGTGGCGACAACGCTGAAACCTACACCGGTGGTCTGAAATACGACG \\
\hline Protein & 226 & $\begin{array}{llllllllllllllllllllll}\text { G } & \mathrm{D} & \mathrm{Q} & \mathbf{N} & \mathbf{A} & \mathbf{S} & \mathbf{W} & \mathbf{H} & \mathbf{W} & \mathbf{V} & \mathbf{V} & \mathbf{A} & \mathbf{T} & \mathbf{T} & \mathbf{L} & \mathbf{K} & \mathbf{P} & \mathbf{T} & \mathbf{P} & \mathbf{A} & \mathbf{V} & *\end{array}$ \\
\hline K. pneumoniae 2110 & 751 & CCAACAACATCTACCTGGCCACTCAGTACACCCAGACCTACAACGCGACCCGCGCCGGTTCCCTGGGC \\
\hline K. pneumoniae 2554 & 748 & CCAACAACATTTACCTGGCGACTCAGTACACCCAGACTTACAACGCAACTCGTTTCAGCGGCAACGGAGAATCTG \\
\hline K. pneumoniae 2110 & 819 & TTTGCTAACAAAGCGCAGAACTTCGAAGTGGTTGCTCAGTACCAGTTCGACTTCGGTCTGC \\
\hline K. preumoniae 2554 & 823 & ATTCTATTAGCGGTTTTGCTAACAAAGCACAGAACTTCGAAGTGGTTGCTCAGTACCAGTTCGACTTCGGTCTGC \\
\hline K. pneumoniae 2110 & 880 & GTCCGTCCGTGGCTTACCTGCAGTCTAAAGGTAAGGATCTGGAAGGCTACGGCGACCAGGACATCCTGAAATATG \\
\hline K. pneumoniae 2554 & 898 & GTCCGTCCGTAGCTTACCTGCAGTCTAAAGGTAAGGACATCGAAGGTTACGGCGACCAGGACCTGCTGAAATATG \\
\hline K. pneumoniae 2110 & 955 & TTGACGTTGGCGCAACCTACT \\
\hline K. pneumoniae 2554 & 973 & TTGACGTTGGCGCGACCTACT \\
\hline
\end{tabular}

Fig. 4. Nucleotide sequences of the ompK36 gene of $Z 2110$ and $Z 2554$, and the putative protein sequence. The nucleotide sequence of $о m p K 36$ and its translated protein sequence were analysed by DNAMAN and compared with wild-type $K$. pneumoniae C3 (GenBank accession no. Z33506). The locations where Z2110 is different from the sequence of C3 are shown in bold and underlined. Asterisks indicate the location of the stop codons, which are shown in italic and underlined.

We realized that in strain Z2110, although OmpK36 was not expressed, there was an additional protein band of $\sim 26 \mathrm{kDa}$ seen by SDS-PAGE (Fig. 3). An early termination of translation might lead to a truncated protein of $\sim 26 \mathrm{kDa}$ - in other words, we suspected that the new $\sim 26 \mathrm{kDa}$ protein in Z2110 might be the truncated product of OmpK36. If that were the case, then it indicates that the truncated C-terminal portion of the OmpK36 protein plays a critical role in drug resistance. After SDS-PAGE and protein transfer, we analysed the $\mathrm{N}$-terminal amino acid sequence of this protein. The 15 aa determined are shown in Fig. 5. Comparing the sequence of the determined Nterminal sequence with the putative sequence of the ompK36 gene, we were unable to find any similarity, suggesting that the newly discovered $\sim 26 \mathrm{kDa}$ protein from Z2110 was not a truncated OmpK36 protein. To understand the nature of this protein, we searched GenBank to look for potential homologous sequences. We found a hypothetical protein called KPN_03267 originating from K. pneumoniae subsp. pneumoniae MGH 78578 (GenBank accession no. YP_001336895), which showed $100 \%$ similarity to the 15 determined N-terminal amino acids. The sequence of this putative protein is shown in Fig. 5. This protein has 231 aa in total with an approximate molecular mass of $25.9 \mathrm{kDa}$, which is similar to the protein we observed in Z2110. However, transmembrane structure analysis using ConPre II software conducted for this protein revealed that there is no transmembrane topology in this sequence. Therefore, this protein is unable to fold into a transmembrane structure to form a channel, as does OmpK36. It is possible that the replacement of OmpK36 with a new protein of $\sim 26 \mathrm{kDa}$ resulted in the loss of the channel function, which eventually leads to resistance in the strain Z2110 in addition to the high-level expression of different types of $\beta$-lactamases. Currently, the contribution of this new protein to drug resistance in strain Z2110 is unclear and is worthy of further study.

K. pneumoniae contains two major porins, OmpK35 and OmpK36, which correspond to OmpF and OmpC, respectively, in E. coli, and OmpK37, which is not expressed or is expressed at very low levels under 


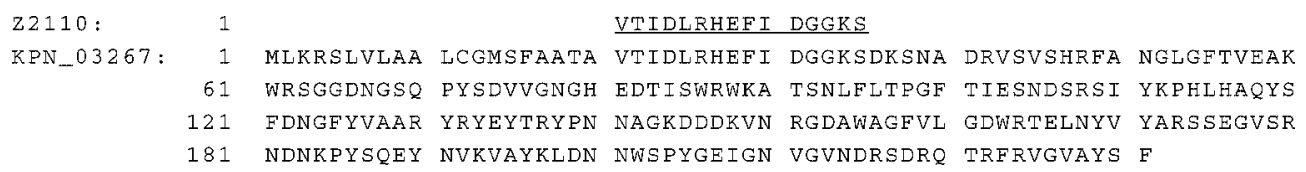

Fig. 5. The $\mathrm{N}$-terminal amino acid sequence of the $\sim 26 \mathrm{kDa}$ protein from $\mathrm{Z} 2110$ compared with the putative amino acid sequence of hypothetical protein KPN_03267 (K. pneumoniae subsp. pneumoniae MGH 78578). The underlined sequence shown at the top is the $\mathrm{N}$-terminal sequence of the protein from Z2110.

laboratory conditions and is undetectable by SDS-PAGE (Doménech-Sánchez et al., 1999). Loss of either OmpK35 or OmpK36 in K. pneumoniae leads to reduced susceptibility to cefoxitin and cefotaxime (MIC $2-4 \mu \mathrm{g} \mathrm{ml}^{-1}$ ), but maintains sensitivity to carbapenems (MIC $0.06-0.125 \mu \mathrm{g}$ $\mathrm{ml}^{-1}$ ). However, loss of both porins can result in reduced susceptibility to imipenem and meropenem (MIC 0.25 $2 \mu \mathrm{g} \mathrm{ml}^{-1}$ ) (Doménech-Sánchez et al., 1999). Loss of one of the porins combined with broad-spectrum $\beta$-lactamase and/or ESBL production in $K$. pneumoniae can cause resistance to cefoxitin and cephalosporin whilst maintaining susceptibility to imipenem (Martínez-Martínez et al., 1996; Rice et al., 1993), whilst combining with AmpC $\beta$ lactamase expression results in resistance or reduced susceptibility to carbapenems (Bradford et al., 1997; Cao et al., 2000; Lee et al., 2007b). This view is supported by the observations in K. pneumoniae Z2110 in the current study. Loss of both porins combined with broad-spectrum $\beta$ lactamase and/or ESBL production would result in moderate resistance or reduced susceptibility to imipenem and meropenem, as well as resistance to ertapenem (Crowley et al., 2002; Elliott et al., 2006; Jacoby et al., 2004). Lee et al. (2007a) reported that $K$. pneumoniae isolates Lkp11-13 that produced CTX-M-3 and SHV-5 but with OmpK35 deficiency and OmpK36 expression were susceptible to carbapenems. Genetically related K. pneumoniae Lkp14 producing an extra DHA-1 and failing to express OmpK36 showed resistance to ertapenem, and reduced susceptibility to imipenem and meropenem. The introduction of a $b l a_{\mathrm{DHA}-1}$-carrying plasmid into Lkp11 only resulted in a significant increase in the MICs of cephamycins and $\beta$-lactamase inhibitors, and a slight increase in the MIC of ertapenem, but not in those of imipenem and meropenem. Mena et al. (2006) also reported that CTX-M-1-producing $K$. pneumoniae that expressed one of the two major porins, OmpK36, was susceptible to carbapenems, whereas isolates that did not express either of the porins were resistant to carbapenems. In this study, two clinical isolates of genetically unrelated, OmpK36-deficient, OmpK35-expressing K. pneumoniae were investigated. K. pneumoniae Z2110 producing TEM1, CTX-M-14 and plasmid-mediated DHA- $1 \beta$-lactamase was resistant to ertapenem, and had reduced susceptibility to imipenem and meropenem as expected. K. pneumoniae Z2554 producing TEM-1 and CTX-M-14 but not DHA-1, however, showed a similar resistance phenotype for carbapenems. Thus, we believe that OmpK36 may play an important role in the resistance or reduced susceptibility to carbapenems in $K$. pneumoniae isolates producing AmpC, ESBL or broad-spectrum $\beta$-lactamase. To the best of our knowledge this is the first report of clinical isolates of $K$. pneumoniae with reduced carbapenem susceptibility that lacked OmpK36 but showed expression of OmpK35, and provides further evidence that OmpK36 may be more important than OmpK35 in resistance.

Jacoby et al. (2004) reported that most porin-deficient $K$. pneumoniae derivatives producing plasmid-mediated $\beta$ lactamases are resistant to ertapenem but have only slightly reduced susceptibility to imipenem and meropenem. A similar carbapenem efficacy for porin-deficient K. pneumoniae clinical isolates was observed in the current study. In summary, we have described two clinical isolates of $K$. pneumoniae deficient in OmpK36 with resistance to ertapenem and reduced susceptibility to imipenem and meropenem, and have provided a detailed molecular analysis of their antimicrobial drug resistance mechanisms. Expression of OmpK35 was not sufficient to recover resistance to ertapenem or the reduced susceptibility to imipenem and meropenem in these K. pneumoniae isolates, suggesting that OmpK36 plays an important role in the resistance or reduced susceptibility to carbapenems in $K$. pneumoniae isolates producing AmpC, ESBL or broadspectrum $\beta$-lactamase.

\section{REFERENCES}

Aktas, Z., Bal, C., Midilli, K., Poirel, L. \& Nordmann, P. (2006). First IMP-1-producing Klebsiella pneumoniae isolate in Turkey. Clin Microbiol Infect 12, 695-696.

Albertí, S., Rodríquez-Quinoñes, F., Schirmer, T., Rummel, G., Tomás, J. M., Rosenbusch, J. P. \& Benedi, V. J. (1995). A porin from Klebsiella pneumoniae: sequence homology, three-dimensional model, and complement binding. Infect Immun 63, 903-910.

Bradford, P. A., Urban, C., Mariano, N., Projan, S. L., Rahal, J. J. \& Bush, K. (1997). Imipenem resistance in Klebsiella pneumoniae is associated with the combination of ACT-1, a plasmid-mediated AmpC $\beta$-lactamase, and the loss of an outer membrane protein. Antimicrob Agents Chemother 41, 563-569.

Cai, J. C., Zhou, H. W., Zhang, R. \& Chen, G. X. (2008). Emergence of Serratia marcescens, Klebsiella pneumoniae and Escherichia coli possessing the plasmid-mediated carbapenem-hydrolyzing $\beta$-lactamase KPC-2 in intensive care units of a Chinese hospital. Antimicrob Agents Chemother 52, 2014-2018. 
Cao, V. T., Arlet, G., Ericsson, B. M., Tammelin, A., Courvalin, P. \& Lambert, T. (2000). Emergence of imipenem resistance in Klebsiella pneumoniae owing to combination of plasmid-mediated CMY-4 and permeability alteration. J Antimicrob Chemother 46, 895-900.

CLSI (2006). Methods for Dilution Antimicrobial Susceptibility Tests for Bacteria that Grow Aerobically, approved standard M7-A7. Wayne, PA: Clinical and Laboratory Standards Institute.

Crowley, B., Benedí, V. J. \& Doménech-Sánchez, A. (2002). Expression of SHV-2 $\beta$-lactamase and of reduced amounts of OmpK36 porin in Klebsiella pneumoniae results in increased resistance to cephalosporins and carbapenems. Antimicrob Agents Chemother 46, 3679-3682.

Doménech-Sánchez, A., Hernández-Allés, S., Martínez-Martínez, L., Benedí, V. J. \& Albertí, S. (1999). Identification and characterization of a new porin gene of Klebsiella pneumoniae: its role in $\beta$-lactam antibiotic resistance. J Bacteriol 181, 2726-2732.

Doménech-Sánchez, A., Martínez-Martínez, L., Hernández-Allés, S., Conejo, M. C., Pascual, A., Tomás, J. M., Albertí, S. \& Benedí, V. J. (2003). Role of Klebsiella pneumoniae OmpK35 porin in antimicrobial resistance. Antimicrob Agents Chemother 47, 3332-3335.

Elliott, E., Brink, A. J., van Greune, J., Els, Z., Woodford, N., Turton, J., Warner, M. \& Livermore, D. M. (2006). In vivo development of ertapenem resistance in a patient with pneumonia caused by Klebsiella pneumoniae with an extended-spectrum $\beta$-lactamase. Clin Infect Dis 42, e95-e98.

Hernández-Allés, S., Albertí, S., Alvarez, D., Doménech-Sánchez, A., Martínez-Martínez, L., Gil, J., Tomás, J. M. \& Benedi, V. J. (1999). Porin expression in clinical isolates of Klebsiella pneumoniae. Microbiology 145, 673-679.

Jacoby, G. A., Mills, D. M. \& Chow, N. (2004). Role of $\beta$-lactamases and porins in resistance to ertapenem and other $\beta$-lactams in Klebsiella pneumoniae. Antimicrob Agents Chemother 48, 3203-3206.

Kaczmarek, F. M., Dib-Hajj, F., Shang, W. \& Gootz, T. D. (2006). High-level carbapenem resistance in a Klebsiella pneumoniae clinical isolate is due to the combination of $b{ }_{\mathrm{ACT}-1} \beta$-lactamase production, porin OmpK35/36 insertional inactivation, and down-regulation of the phosphate transport porin PhoE. Antimicrob Agents Chemother 50, 3396-3406.

Koh, T. H., Babini, G. S., Woodford, N., Sng, L. H., Hall, L. M. \& Livermore, D. M. (1999). Carbapenem-hydrolysing IMP-1 $\beta$-lactamase in Klebsiella pneumoniae from Singapore. Lancet 353, 2162.

Lee, C. H., Chu, C., Liu, J. W., Chen, Y. S., Chiu, C. J. \& Su, L. H. (2007a). Collateral damage of flomoxef therapy: in vivo development of porin deficiency and acquisition of $b l a_{\mathrm{DHA}-1}$ leading to ertapenem resistance in a clinical isolate of Klebsiella pneumoniae producing CTX-M-3 and SHV-5 $\beta$-lactamases. J Antimicrob Chemother 60, 410413

Lee, K., Yong, D., Choi, Y. S., Yum, J. H., Kim, J. K., Woodford, N., Livermore, D. M. \& Chong, Y. (2007b). Reduced imipenem susceptibility in Klebsiella pneumoniae clinical isolates with plasmidmediated CMY-2 and DHA-1 $\beta$-lactamases co-mediated by porin loss. Int J Antimicrob Agents 29, 201-206.

Lincopan, N., McCulloch, J. A., Reinert, C., Cassettari, V. C., Gales A. C. \& Mamizuka, E. M. (2005). First isolation of metallo- $\beta$ lactamase-producing multiresistant Klebsiella pneumoniae from a patient in Brazil. J Clin Microbiol 43, 516-519.

Macrina, F. L., Kopecko, D. J., Jones, K. R., Ayers, D. J. \& McCowen S. M. (1978). A multiple plasmid-containing Escherichia coli strain: convenient source of size reference plasmid molecules. Plasmid 1, $417-420$.
Martínez-Martínez, L., Hernández-Allés, S., Albertí, S., Tomás, J. M., Benedi, V. J. \& Jacoby, G. A. (1996). In vivo selection of porindeficient mutants of Klebsiella pneumoniae with increased resistance to cefoxitin and expanded-spectrum cephalosporins. Antimicrob Agents Chemother 40, 342-348.

Mathew, A., Harris, A. M., Marshall, M. J. \& Ross, G. W. (1975). The use of analytical isoelectric focusing for detection and identification of $\beta$-lactamase. J Gen Microbiol 88, 169-178.

Mena, A., Plasencia, V., Garcia, L., Hidalgo, O., Ayestarán, J. I., Alberti, S., Borrell, N., Pérez, J. L. \& Oliver, A. (2006). Characterization of a large outbreak by CTX-M-1-producing Klebsiella pneumoniae and mechanisms leading to in vivo carbapenem resistance development. J Clin Microbiol 44, 2831-2837.

Rice, L. B., Carias, L. L., Etter, L. \& Shlase, D. M. (1993). Resistance to cefoperazone-sulbactam in Klebsiella pneumoniae: evidence for enhanced resistance resulting from the coexistence of two different resistance mechanisms. Antimicrob Agents Chemother 37, 1061-1064.

Smith Moland, E., Hanson, N. D., Herrera, V. L., Black, J. A., Lockhart, T. J., Hossain, A., Johnson, J. A., Goering, R. V. \& Thomson, K. S. (2003). Plasmid mediated, carbapenem-hydrolysing $\beta$-lactamase, KPC-2, in Klebsiella pneumoniae isolates. J Antimicrob Chemother 51, 711-714.

Tenover, F. C., Arbeit, R. D., Goering, R. V., Mickelsen, P. A., Murray, B. E., Persing, D. H. \& Swaminathan, B. (1995). Interpreting chromosomal DNA restriction patterns produced by pulsed-field gel electrophoresis: criteria for bacterial strain typing. J Clin Microbiol 33, 2233-2239.

Wei, Z. Q., Du, X. X., Yu, Y. S., Shen, P., Chen, Y. G. \& Li, L. J. (2007). Plasmid-mediated KPC-2 in a Klebsiella pneumoniae isolate from China. Antimicrob Agents Chemother 51, 763-765.

Woodford, N., Tierno, P. M., Young, K., Jr, Tysall, L., Palepou, M.-F. I., Ward, E., Painter, R. E., Suber, D. F., Shungu, D. \& other authors (2004). Outbreak of Klebsiella pneumoniae producing a new carbapenem-hydrolyzing class A $\beta$-lactamase, KPC-3, in a New York medical center. Antimicrob Agents Chemother 48, 4793-4799.

Yan, J. J., Ko, W. C., Tsai, S. H., Wu, H. M. \& Wu, J. J. (2001). Outbreak of infection with multidrug-resistant Klebsiella pneumoniae carrying $b l a_{\mathrm{IMP}-8}$ in a university medical center in Taiwan. J Clin Microbiol 39, 4433-4439.

Yan, J. J., Ko, W. C., Jung, Y. C., Chuang, C. L. \& Wu, J. J. (2002). Emergence of Klebsiella pneumoniae isolates producing inducible DHA-1 $\beta$-lactamase in a university hospital in Taiwan. $J$ Clin Microbiol 40, 3121-3126.

Yigit, H., Queenan, A. M., Anderson, G. J., Doménech-Sánchez, A., Biddle, J. W., Steward, C. D., Albertí, S., Bush, K. \& Tenover, F. C. (2001). Novel carbapenem-hydrolyzing $\beta$-lactamase, KPC-1, from a carbapenem-resistant strain of Klebsiella pneumoniae. Antimicrob Agents Chemother 45, 1151-1161.

Yu, Y., Ji, S., Chen, Y., Zhou, W., Wei, Z., Li, L. \& Ma, Y. (2007). Resistance of strains producing extended-spectrum $\beta$-lactamases and genotype distribution in China. J Infect 54, 53-57.

Zhang, R., Zhou, H. W., Cai, J. C. \& Chen, G. X. (2007). Plasmidmediated carbapenem-hydrolysing $\beta$-lactamase KPC-2 in carbapenem-resistant Serratia marcescens isolates from Hangzhou, China. J Antimicrob Chemother 59, 574-576.

Zhang, R., Yang, L., Cai, J. C., Zhou, H. W. \& Chen, G. X. (2008). Highlevel carbapenem resistance in a Citrobacter freundii clinical isolate is due to a combination of KPC-2 production and decreased porin expression. J Med Microbiol 57, 332-337. 